\title{
Easy Breathing-Definition of a Gamification System to Support the Chronic Care of Childhood Asthma
}

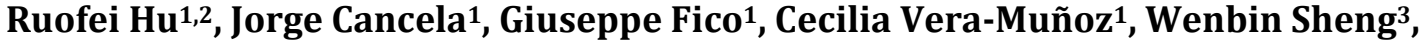 \\ Maria Teresa Arredondo ${ }^{1}$ \\ ${ }^{1}$ Lifestyle Supporting Technologies Group, Technical University of Madrid, Madrid, Spain \\ ${ }^{2} \mathrm{R} \&$ \& D Department, Easy-Benefit Science and Technology Ltd., Hangzhou, China \\ ${ }^{3}$ Pediatrics Department, Hangzhou First People’s Hospital, Hangzhou, China \\ Email: rhu@lst.tfo.upm.es, jcancela@lst.tfo.upm.es, gfico@lst.tfo.upm.es, cvera@lst.tfo.upm.es, swb8825@163.com,mta@lst.tfo.upm
}

How to cite this paper: Hu, R.F., Cancela, J., Fico, G., Vera-Muñoz, C., Sheng, W.B. and Arredondo, M.T. (2016) Easy Breathing -Definition of a Gamification System to Support the Chronic Care of Childhood Asthma. J. Biomedical Science and Engineering, 9, 122-128.

http://dx.doi.org/10.4236/jbise.2016.910B016

Received: August 19, 2016

Accepted: September 20, 2016

Published: September 23, 2016

\begin{abstract}
Asthma is the most common noncommunicable disease among children. It leads to substantial problems both in health and economic terms to individuals and families. This paper introduces a technological solution, Easy Breathing, that supports the management of childhood asthma, providing tools for both doctors and patients. Through the establishment of an asthma chronic care model, it combines doctors' professional guidance and a gamification system to improve the compliance of patients with their treatments and to provide them with tools for the self-management of their disease. The system is in the testing phase, the first mockup has been developed and validated with 270 medical experts and patients, and it will be tested with 540 end-users over the next months.
\end{abstract}

\section{Keywords}

Childhood Asthma, Gamification, Serious Games, Chronic Care-Model

\section{Introduction}

Asthma is a heterogeneous disease, usually characterized by chronic airway inflammation. It is defined by the history of respiratory symptoms such as wheeze, shortness of breath, chest tightness and cough that vary over time and in intensity, together with variable expiratory airflow limitation. The global asthma report informs that $14 \%$ of worldwide children showed asthmatic symptoms in 2013 [1]. Asthma can harm children's health badly and it could lead to lifetime health problems for children, increasing 
the risk of developing other diseases and disabilities, even engendering social and psychological problems later in life [2]. Asthma can also generate a vast economic burden as is reflected in a significant number of scientific studies [3]. The economic costs of asthma are ranked as one of the highest among chronic diseases [3]. Thus, a solution to reduce the incidence and effects of childhood asthma could be conducive to the patient's health and the family's financial condition.

According to the Global Initiative for Asthma (GINA) guidelines, long-term asthma management requires a combination of pharmacological and non-pharmacological strategies. A common approach for all patients with asthma includes: correct inhaler skills and adherence to the medication use, a written asthma action plan for worsening or attack, advice about self-monitoring of symptoms and peak expiratory flow (PEF), and regular medical review [4].

In any case, the effective management of asthma requires the patient's cooperation outside of the hospital. Information and Communication Technologies (ICTs) can provide tools for both professionals and patients to let patients or their family members access the professional services anytime and anywhere. New sensors, together with communication and processing capabilities, can make the collection, storage, analysis and transmission of patients' data much easier than years ago. ICTs can contribute a tool to implement an asthma care system based on the global asthma management guidelines to create a professional and personalized asthma care plan for each patient. Particularly, this work describes an eHealth application, Easy Breathing, to support the self-management of children and their parents for childhood asthma. It allows patients to get the remote professional and appropriate care by connecting them with their doctors, educating children and parents, generating a personalized manage plan, providing mechanisms for the self-management, and alerting about potential risky situations.

\section{Methods and Materials}

\subsection{Methods}

This work was organized in three phases: first, existing asthma treatment and management methods were analyzed, whilst related existing technological solutions were also explored; secondly, a childhood asthma care model was defined and integrated into the first prototype of the Easy Breathing system, which was iteratively updated with the involvement of experts and patients; finally, a first version of the Easy Breathing App was developed.

1) State of the Art

A literature review and four expert interviews with three respiratory disease specialists were carried out in order to gain in-depth understanding of the causes and consequences of childhood asthma, and its prevention and treatment solutions. In parallel, a research on technological solutions to support these processes was also carried out, in order to support the design of the system architecture.

2) Care Model Definition

53 experts and 58 patients were interviewed to elicit the users' needs and to define 
the system requirements for providing a service for the management of all asthma dimensions. As part of this process, the result of the state of the art study was used to establish a childhood asthma care model as a reference for the prototype design.

3) Prototype Design

Early prototyping was used within this work as a way to start a dialogue with experts and users and to obtain their feedback. The prototype was designed according to the asthma care model. Then, it was shown and discussed with 20 respiratory disease specialists to evaluate the functionalities, and determine if the system was able to provide support for every important dimension of the treatment. It was also presented to 250 patients to evaluate their acceptance. The prototype was constantly redefined and iteratively updated. Six face to face interviews and three phone call interviews with test users were done for the system optimization. Another 2 meetings with the asthma experts were organized before the development step. The iteration of the prototype was repeated for 14 times.

4) App Development

The final prototype was developed as a App supporting both Android and iOS platforms that will be used in the next validation phase involving more patients and professionals.

\subsection{Materials}

1) Axure RP Pro

Axure RP Pro is an application designed for rapid prototyping, allowing the integration of comments, notes, flow charts, frames and other elements to obtain a complete product presentation. The outcomes of the care model definition, the system framework and function modules, and the first prototype were designed and visualized through Axure.

2) IDEA and Xcode

IntelliJ IDEA (IDEA) was used for the development of the Android version and Xcode for the iOS version.

\section{Results}

\subsection{The Baseline}

The long-term goals for efficient asthma management are symptom control and risk reduction. The disease self-management requires: information, inhaler skills, adherence, written asthma action plan, self-monitoring, and regular reviews [4]. The research carried out on the existing ICTs solutions used these 6 basic dimensions to evaluate their advantages and disadvantages. The existing ICTs systems applied different strategies to different asthma management dimensions: multimedia and interactive instructions can help parents and children to learn about asthma management aspects in an easier and more interesting way (e.g. basic logic of the diagnosis and treatment, and the correct usage of the inhaler medication and peak flow meter). Also, some warning systems can detect the patients' unusual state and provide recommendations according 
to the asthma action plan. Finally, the monitor and alert system can motivate the patients to realize the long-term self-monitoring and improve the medication compliance, and promote the regular review. Nevertheless, all of them lack the provision of a personalized service according to the patient's specific situation, such as age, life style, disease duration, risk factors (e.g. allergies, physical activity), asthma control state and severity, etc.

\subsection{Asthma Care Model}

According to the medical experts' opinion and asthma guidelines, an effective asthma management is contingent upon the state of the patient and the strategy to follow in each case will be different, as shown in Table 1.

\subsection{Definition of Easy Breathing System}

According to the asthma care model, an ICT system called Easy Breathing was defined as the following.

1) System Structure

Easy Breathing is a two-ends system involving both patients (or patients' family members) and doctors. As shown in Figure 2, patients and doctors use the smart devices to realize some online communication and operation, together with the direct contact offline, to realize the treatment during the whole disease course.

2) Easy Breathing's Solution

The Easy Breathing's functional modules are defined according to the childhood asthma care factors. Education module: provides disease knowledge, inhaled drug and PEF meter's operating instruction video, and other disease related information for all patients. It also generates a personalized education plan according to the medical history of each patient and the risk factors. Medication compliance module: generates reminders according to doctor's advice. Symptom and PEF monitoring module: provides an asthma diary to help patients recording the symptoms and PEF values. A smart

Table 1. Childhood asthma care factors.

\begin{tabular}{ll}
\hline \multicolumn{1}{c}{ State } & Action \\
\hline Daily management & Medication compliance \\
& Symptom and PEF monitoring \\
& Regular medical review \\
& Lifestyle modification \\
& Risk factor avoiding \\
Worsening or attack & Early attack warning \\
& Action plan recommendation \\
& Fast medical resources accessing \\
\hline
\end{tabular}


PEF meter can make the data collecting easier. Regular medical review module: generates a weekly evaluation and provides the doctor's manual evaluation every 4 weeks to update patient's control state regularly. Lifestyle modification module: generates the lifestyle tasks of nutrition, physical activity and work and rest adjust according to the patients' control state. Risk factor avoiding module: classifies patients according to their risk factors, and provides the risk avoiding recommendations based on the classification. Early attack warning module: real-time attack alerts based on the PEF value, symptoms collection, medication takings, environment data, allergen contact and exercise level. Action plan advising module: updates the personalized action plan when detecting the patients' asthma worsening or an attack. Fast medical resources accessing module: provides the online-consulting and off-line medical arrangement for the minor abnormal situations. The system will recommend the patients to directly access the emergency center in serious abnormal situations.

To achieve a long-term engagement and motivation, a gamification mechanism was implemented in the Easy Breathing. The system generates a personalized "to do" list for each patient, containing a point system (experience point and coin point), when patient uses the App, he/she will get points. These experience points are related to the user level that granted access to privileges. The collected coins can be exchanged for medical services. User ranking is also used in the system design, so that patients can know their asthma control and their self-management rank among peers. An online patient community is also created to allow patients to make more interaction in the system.

3) Easy Breathing's Prototype

Once the Easy Breathing system was defined, the first prototype was designed based on the described functional modules, and, then, it was continuously reviewed and iteratively updated. Easy Breathing was provided to children aged 5 to 14 . The patients end contains: personal asthma file, personalized asthma management tasks plan, disease assessment and early warning, asthma diary, medication reminder, childhood asthma control test (C-ACT), patients community, information, online consult and offline medical arrangement. The home page and part function pages of the patients end are shown in Figure 1.

The doctors end contains: online patient follow up and inquiry tools, multi member cooperation tools, patients' data management tools and asthma disease knowledge base. The home page and part function pages of the doctors end are shown in Figure 2.

Easy Breathing also includes a smart sensor (Figure 3) for the patients to simplify their daily management. It can measure patients' PEF and forced expiratory volume in one second (FEV1) value, and provides realtime feedback, when the value is abnormal, patients and his/her doctor will be warned immediately.

\section{Discussion}

In this paper, the state of the art of the childhood asthma care support was analyzed, a care model was defined, and the Easy Breathing system designed. A prototype of the system was created and iteratively updated to develop the Easy Breathing App on 


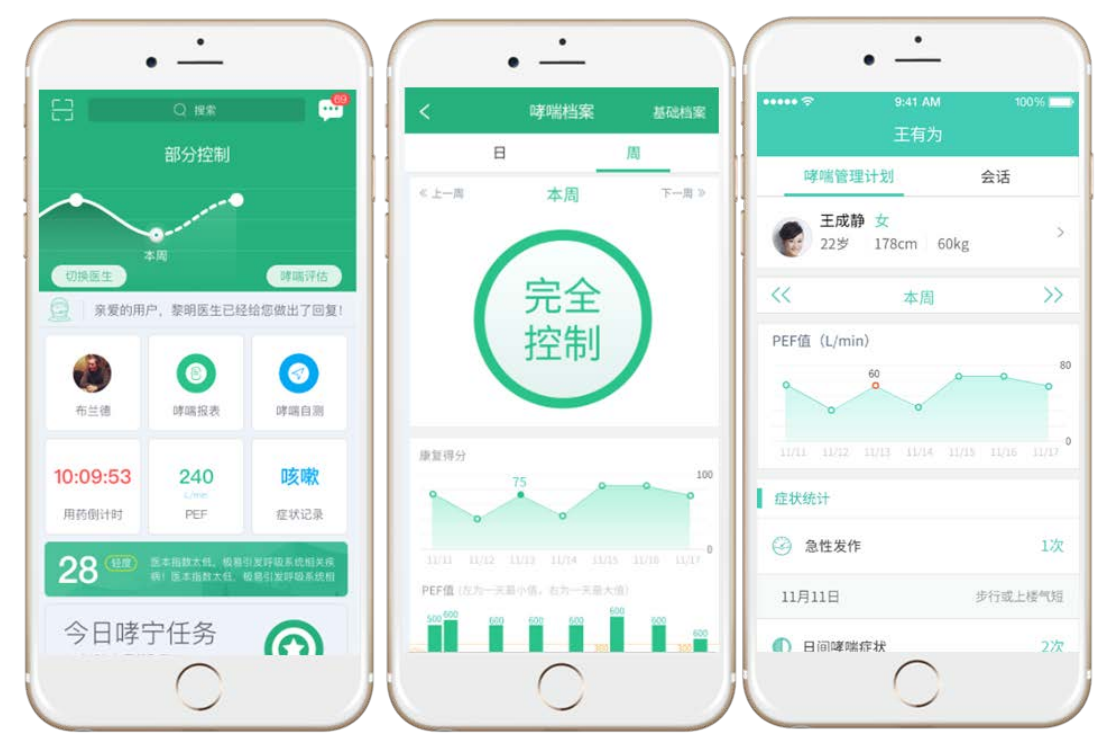

Figure 1. The easy breathing patient end.
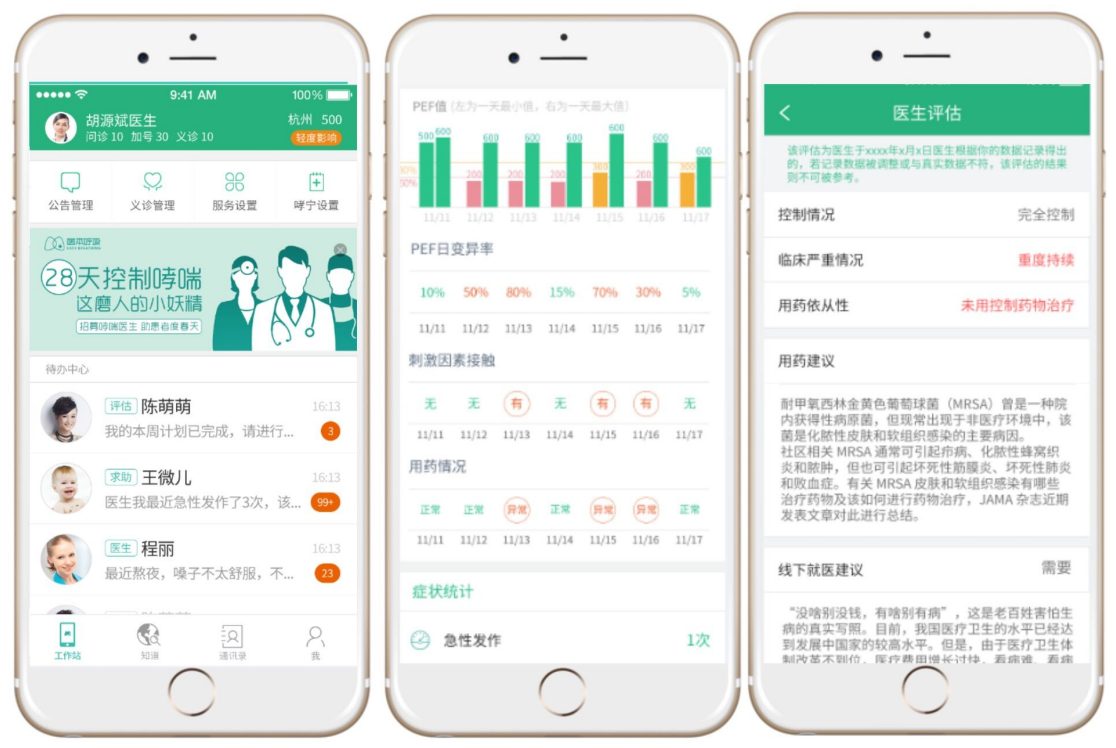

Figure 2. The easy breathing doctor end.

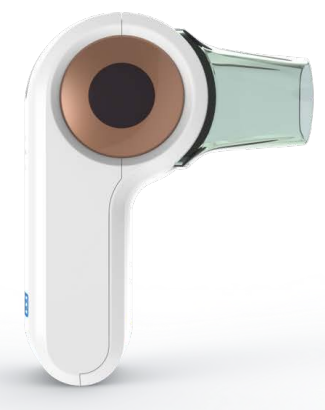

Figure 3. The easy breathing PEF meter. 
Android and iOS platforms. Easy Breathing was tested with a total of 250 patients and 20 doctors. The objective for the next months is to validate the App with at least 500 patients, and updating it according to the feedback received. Particularly, in two different lines: 1) improving and including the gamification mechanisms within the App to increase the adherence and the engagement of the patient, and 2) enhancing the sensors for data collection.

\section{Conclusion}

Management of childhood asthma is a complex problem that requires the cooperation of medical professionals, patients and family members. The Easy Breathing system has been designed to support them in the disease management, by means of a tool aimed at increasing the compliance of patients with the treatment and self-management. This will improve the clinical outcomes, as the system tackles the major aspects for failure of most of the plans for patients: the unfamiliarity with the disease and noncompliance to treatment. Easy Breathing has a smart education system to provide different patients the most suitable knowledge, as well as recommendations or solution for some specific situations. It also includes a gamification mechanism to strengthen the patients' initiative to participate in the management more actively.

\section{References}

[1] ISAAC (2014) The Global Asthma Report 2014. http://www.globalasthmareport.org/burden/burden.php

[2] Moola, F.J., Faulkner, G.E.J., White, L., et al. (2014) The Psychological and Social Impact of Camp for Children with Chronic Illnesses: A Systematic Review Update. Child: Care, Health and Development, 40, 615-631. http://dx.doi.org/10.1111/cch.12114

[3] Bahadori, K., Doyle-Waters, M.M., Marra, C., et al. (2009) Economic Burden of Asthma: A Systematic Review. BMC Pulmonary Medicine, 9, 1.

http://dx.doi.org/10.1186/1471-2466-9-24

[4] GINA (2016) 2016 at a Glance Asthma Scholar. http://ginasthma.org/gina-reports/ 
Submit or recommend next manuscript to SCIRP and we will provide best service for you:

Accepting pre-submission inquiries through Email, Facebook, LinkedIn, Twitter, etc.

A wide selection of journals (inclusive of 9 subjects, more than 200 journals)

Providing 24-hour high-quality service

User-friendly online submission system

Fair and swift peer-review system

Efficient typesetting and proofreading procedure

Display of the result of downloads and visits, as well as the number of cited articles

Maximum dissemination of your research work

Submit your manuscript at: http://papersubmission.scirp.org/

Or contact jbise@scirp.org 